\title{
A Rare Case of Posterior Communicating Artery Pseudoaneurysm Caused by Traumatic Head Injury
}

\author{
Hui Yuan Su${ }^{1}$, Yu-Feng Su ${ }^{1,2}$ and Chih Jen Wang ${ }^{1,3 *}$
}

${ }^{1}$ Department of Surgery, Kaohsiung Medical University Hospital, Taiwan, PR china

${ }^{2}$ Institute of Clinical Medicine, Kaohsiung Medical University, Kaohsiung, Taiwan, PR china

${ }^{3}$ Department of Surgery, College of Medicine, Kaohsiung Medical University, Kaohsiung, Taiwan, PR china

\begin{abstract}
Traumatic intracranial aneurysm is rare and mostly due to blunt head injury. High mortality rate up to $50 \%$ is reported in the literature. Early diagnosis and appropriate management is essential for this fatal disease. We present a rare case of pseudoaneurysm over left-side posterior communicating artery after traumatic head injury. Avulsion of the posterior communicating artery was noted intra-operatively and then clipping of the proximal stump was arranged. There was no delayed hemorrhage after the operation. After review of the literature, there is no definite management for this kind of traumatic intracranial pseudoaneurysm. Here, we present the fourth case of posterior communicating artery traumatic pseudoaneurysm, which presents appropriate outcome after surgical intervention of clipping proximal stump of the avulsed artery.
\end{abstract}

Keywords: Pseudoaneurysm; Posterior communicating artery; Traumatic head injury

\section{Introduction}

Traumatic Intracranial Aneurysms (TICAs) comprise less than $1 \%$ of all intracranial aneurysms. TICAs are formed due to blunt, penetrating, or iatrogenic trauma. TICAs due to blunt injuries are the most common causes [1]. TICAs are associated with a significant mortality of up to $50 \%$. Therefore, early diagnosis and prompt intervention are necessary [2].

\section{Case Report}

A 27-year-old female without systemic disease suffered from traumatic Subarachnoid Hemorrhage $(\mathrm{SAH})$ in a traffic accidence. She was a motorcycle rider with helmet protection. According to the witness, she was collided with the engineering vehicle which was parked by the road and beyond the line. At first, she was taken to a local hospital and then transferred to our hospital under impression of diffused traumatic SAH (Figure 1). At our emergency department, initial vital signs were Glasgow Coma Scale (GCS) E1VTM3 (status post endotracheal intubation), respiratory rate 20 times per minute, blood pressure 104/58 $\mathrm{mmHg}$, pulse rate 116 beat per minute and body temperature 37 degree celsius. Computed tomography and angiography (CTA) was arranged under impression of spontaneous SAH. There was no evidence of cerebral aneurysm (Figure 2). Intracranial pressure monitor was implanted and showed high intra-cranial pressure 37-

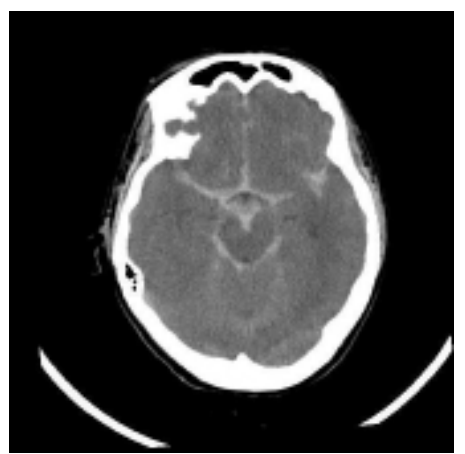

Figure 1: Brain computed tomography shows diffused subarachnoid hemorrhage.

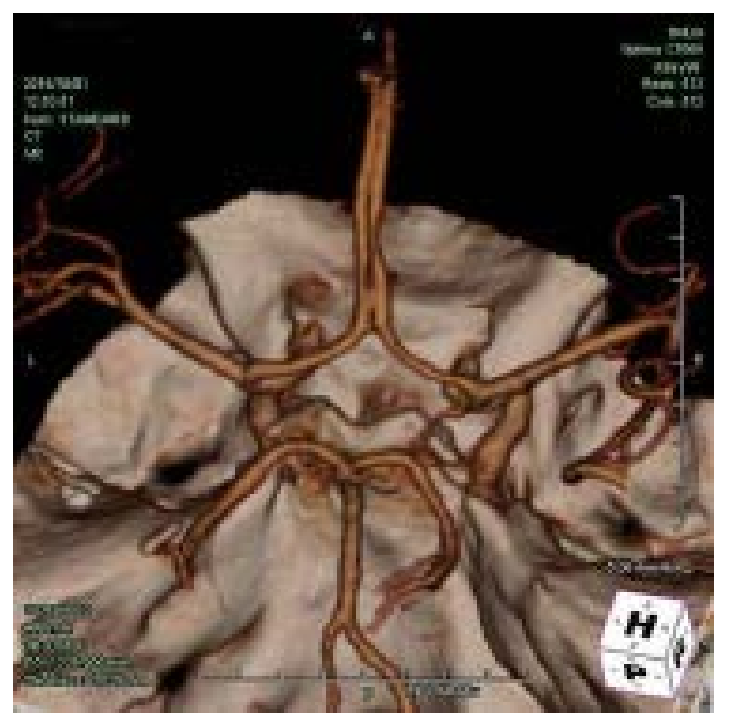

Figure 2: Brain computed tomography shows no evidence of intracranial aneurysms.

$41 \mathrm{mmHg}$. After the operation, she was admitted to our neurosurgery intensive care unit (ICU). Full sedation, mannitol, $3 \% \mathrm{NaCl}$ and hypothermia therapy were all performed but increased intra-cranial pressure (IICP) was still noted. Brain-computed tomography (CT) was arranged and showed multiple brain infarctions over bilateral frontal lobe and right temporal lobe and progression of brain edema (Figure 3).

*Corresponding author: Chih Jen Wang, Department of Surgery, Kaohsiung Medical University Hospital, No. 100, Tzyou 1st Rd., Kaohsiung 807, Taiwan, PR China, Tel: 886-7-3215049; E-mail: ed0116@hotmail.com

Received March 24, 2015; Accepted April 24, 2015; Published April 27, 2015

Citation: Su HY, Su YF, Wang CJ (2015) A Rare Case of Posterior Communicating Artery Pseudoaneurysm Caused by Traumatic Head Injury. J Trauma Treat 4: 248. doi:10.4172/2167-1222.1000248

Copyright: (c) $2015 \mathrm{Su} \mathrm{H}$, et al. This is an open-access article distributed under the terms of the Creative Commons Attribution License, which permits unrestricted use, distribution, and reproduction in any medium, provided the original author and source are credited. 


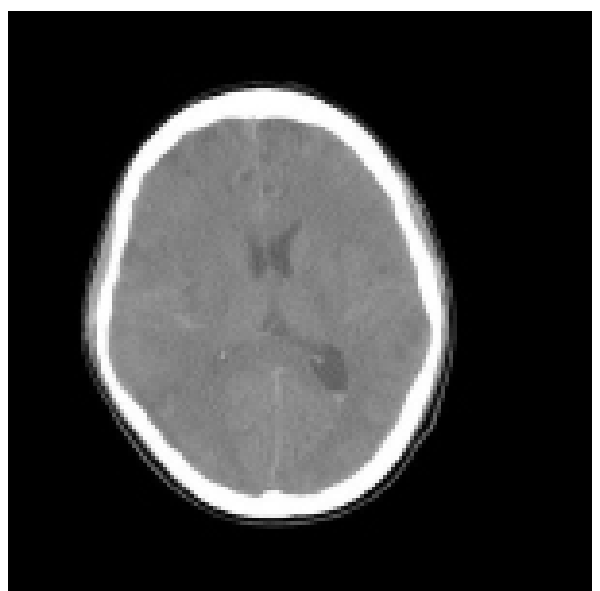

Figure 3: Brain computed tomography shows multiple brain infarction over bilateral frontal lobe and right temporal lobe. Besides, progression of brain edema is also noted.

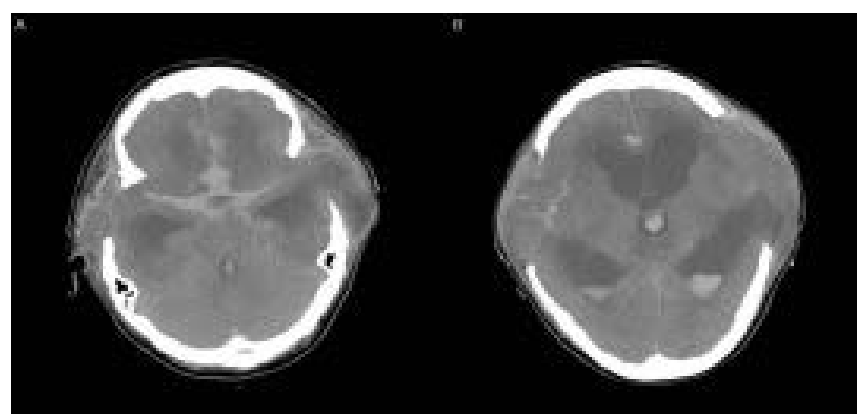

Figure 4A and 4B: Brain computed tomography shows status post bilateral temporo-parietal craniotomy with bilateral transcalvarial brain herniation, interval development of hemoventricle with severe hydrocephalus and worsening of subarachnoid hemorrhage involving the basal cistern and along the bilateral cerebral sulci.

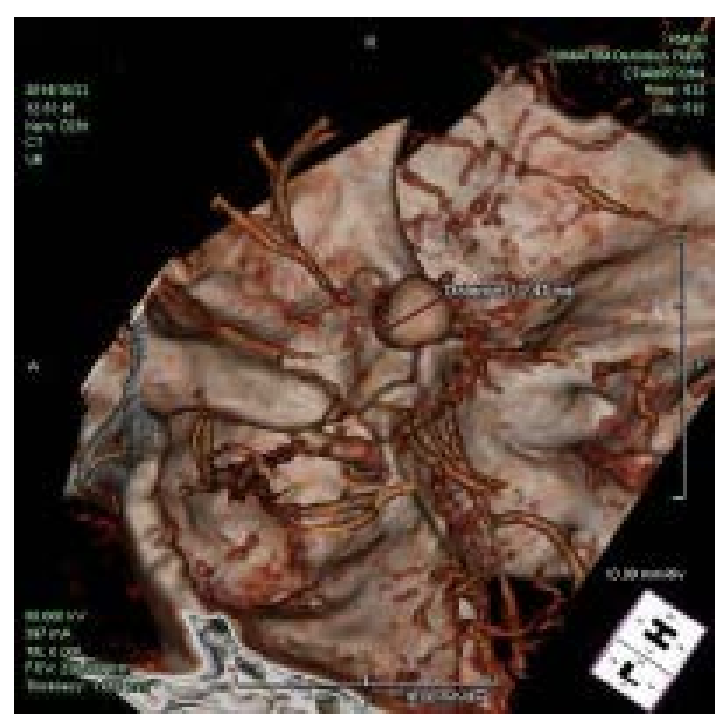

Figure 5A and 5B: There is a huge out-pouching contrast-filling lesion located in the supraclinoid segment of the right internal carotid artery. Diffuse small caliber of intracranial arteries is noted. A huge pseudoaneurysm in the right supraclinoid internal carotid artery is suspected.
Emergent operation of bilateral fronto-temporo-parietal craniectomy was arranged. After that, intra-cranial pressure (ICP) around 14-21 $\mathrm{mmHg}$ was noted.

Furthermore, ICP monitor was removed due to stable condition on ICU day 16. Regular bedside trans-cranial Doppler performed on ICU day 19 showed progression of hydrocephalus, with left middle cerebral artery and right posterior cerebral artery pulsatility index more than 1.2. Under impression of hydrocephalus-related IICP, emergent brain CT was arranged and showed persistent basal cistern $\mathrm{SAH}$ and hydrocephalus (Figure 4A and 4B). Emergent operation of extraventricle drainage tube insertion was conducted for hydrocephalus. Post-operation ICP showed 9-11 mmHg. We also followed CTA due to persistent SAH subsequently. The image showed a large aneurysm with maximum diameter $1.24 \mathrm{~cm}$ (Figure 5A and 5B). For further evaluation, angiography was arranged and showed a huge out-pouching contrastfilling lesion located in the supraclinoid segment of the right internal carotid artery on ICU day 23. After discussion with the family, operation of aneurysm clipping was suggested to performed one month after traumatic head injury. Intra-operatively, active bleeding from ruptured posterior communicating artery (P-com) was noted. Clipping over right side $\mathrm{P}$-com artery was conducted. The post-operation diagnosis of traumatic pseudoaneurysm due to ruptured P-com was confirmed. After that, there was no delayed subarachnoid hemorrhage noted. This patient was transferred to the respiratory care center for weaning off ventilator one month after the operation. The GCS scales was E4VTM3 (status post operation of tracheastomy) before she was transferred to the day-care center for further rehabilitation program.

\section{Discussion}

TICAs are divided into four categories according to the mechanism of injury: following closed head injury, missile injury, penetrating head injury, and iatrogenic injury. The mechanisms causing traumatic aneurysms of supraclinoid internal carotid artery (ICA) are believed to be as follows: Direct injury to the ICA by basal skull fracture, overstretching or torsion of the ICA wall by movement of the brain following impact, tearing by nearby prominent bony structures, and avulsion of a perforator [3]. TICAs are also classified histologically as true or false. True aneurysms are formed after a partial disruption of the arterial wall. The internal elastic lamina and media damages with an intact adventitia form the true aneurysm. The complete penetrating injury of the vessel wall results in the false aneurysm. The hematoma organizes to form the outer wall of the aneurysm. Unlike berry aneurysms, TICAs typically do not have a neck, are more irregular in their dome contour, and are subject to delayed filling and emptying of the sac based on angiograms [4]. On the basis of anatomic location, we divided TICAs secondary to blunt brain injury into two categories: skull base and peripheral TICAs. Furthermore, pseudoaneurysms at the basal skull are classified into infraclinoid, supraclinoid and vertebrobasilar TICAs according to categorization done by Mao et al. [1]. Their anatomical locations reflect the underlying mechanism of injury. For example, infraclinoid ICA and basilar artery aneurysms are associated with basal skull fractures. Besides, supraclinoid ICA aneurysms form as a result of contusion of the artery by the anterior clinoid process or sudden stretching of the artery across the process during impact.

Blunt brain injury could lead to formation of traumatic aneurysms around the cerebral falcine edge and cerebellum tentorium, but not in the trunk or sub-cortical branches of the middle cerebral artery. On the other hand, cortical artery aneurysms develop beneath an overlying skull fracture $[1,2]$. 
In our case, P-com pseudoaneurysm is formed due to disrupted vessel of P-com artery caused by sudden stretching during impact. It is a kind of skull base aneurysm, but it is difficult to be classified into any subgroup according to current categorization. To our knowledge, this is the fourth case report in which P-com traumatic aneurysms are caused by craniocerebral injury.

We review three case of post-traumatic P-com aneurysm. The first case is a 37 -year-old man with a severe head injury after a traffic accident. No mass lesion or SAH in the suprasellar cistern were noted on brain CT. One month after the injury, polydipsia, hyperdiuresis, a right pulsatile exophthalmos, right conjunctival chemosis, and a retroocular bruit synchronous with the patient's pulse gradually developed. CTA revealed a large saccular aneurysm originating from the P-com artery. Besides, coronal and sagittal view of CT showed pituitary stalk compression by the P-com artery aneurysm. Angiography of the right ICA showed an aneurysm was in continuity with the cavernous sinus and could not be completely separated from the supraclinoid ICA or the P-com artery. The right vertebral angiograms revealed that the cavernous sinus fistula was supplied by a large dome-shaped ruptured aneurysm with a diameter of $10 \mathrm{~mm}$ arising from the P-com artery. Endovascular treatment for the aneurysm and aneurysmal cavernous fistula was arranged. After the procedure, angiography demonstrated complete occlusion of the aneurysm and the fistula [5].

The second case is a 46-year-old woman who was passenger in a car that struck a telephone pole 31 years ago. This patient sustained extensive frontal skull and pelvic injuries, which required a 2 -month hospital stay. She noted right exophthalmos 16 years after injury but she did not pay attention on it. Cerebral angiography was performed due to worse condition of exophthalmos. Angiography performed in the lateral projection demonstrated immediate opacification of an aneurysmal P-com artery with decompression into the right cavernous sinus. At a subsequent surgery, a clip was placed occluding the anterior origin of the right P-com artery. Post-operative angiography showed persistent decompress of the P-com artery through the fistula. Further surgical intervention was suggested but this patient refused due to improved condition of exophthalmos [6].

The third case is a 16-year-old boy with a linear fracture in the right frontal base after a traffic accident. He rode a mini-motorcycle and overturned into a ditch. Brain CT revealed SAH over the basal cistern. Day 17 after the accident, angiography was performed due to right eye chemosis. Carotid angiography revealed the carotid cavernous fistula which was associated with an intradural pseudoaneurysm at the junction of ICA and P-com artery, which drained directly into the cavernous sinus. Direct surgery was performed 30 days after the accident. Postoperative angiography confirmed the obliteration of both the aneurysm and the carotid-cavernous fistula [7].

Although CTA has improved significantly over the past few years as a non-invasive screening method to detect intracranial aneurysms, it is not sensitive enough to replace conventional digital subtraction angiography. TICAs rarely regress and they have a high incidence of rupture. Thus, treatment should be instituted once the diagnosis is made. TICAs should be suspected in all patients with severe head injury, especially if presenting with delayed neurologic deterioration [2].

Angiographically, berry aneurysms are most commonly located at the proximal bifurcations of major arteries or their primary branches. Traumatic intracranial aneurysms more commonly involve the arterial wall along the course of secondary branches. Unlike berry aneurysms, TICAs typically do not have a clear neck, are more irregular in their dome contour, and are often observed to be subject to delayed filling and emptying of the sac. Most traumatic aneurysms present in a delayed fashion, ranging from hours to years after the inciting trauma. Rupture is the most common early presentation, bi-modally distributed at $24 \mathrm{hr}$ and 3 weeks after trauma. The time between injury and diagnosis has ranged from within 24 hours to 25 years. More than half of reported cases were diagnosed within 3 weeks $[8,9]$. Angiography performed the day of injury detected traumatic aneurysms in only $54 \%$ of the case. Therefore, angiography should be performed repeatedly, but less risky modalities such as three-dimensional CT (3D-CT) and magnetic resonance angiography can be selected. The presence of diffuse SAH in the basal cistern or repeated SAH indicates angiography or alternative examination such as 3D-CT angiography soon after stabilization. Angiography should be repeated 2 weeks later if no aneurysm is detected, because early angiography results in a large percentage of false negatives. However, repeated monitoring of the size and shape of the aneurysm with 3D-CT or magnetic resonance angiography is absolutely necessary [3]. The total mortality rate is $34.6 \%$. Nonoperative management is associated with a $50 \%$ mortality rate. The reported combined morbidity and mortality rate associated with surgical management is approximately $22 \%[8,9]$.

Most traumatic aneurysms are pseudoaneurysms and have broad necks, so direct clipping or endovascular obliteration is rarely successful [3]. Our case had the typical characteristics of traumatic pseudoaneurysm including wide neck and huge size. There exists a high risk of failure to embolization of the lesion by endovascular intervention. In the literature, most pseudoaneurysms formed at the junction of the supraclinoid IC and P-com. These pseudoaneurysms are accompanied with Carotid Cavernous Fistula (CCF). These cases are treated by transarterial coil embolization, surgical clipping of P-com aneurysm, neck of aneurysm or fistulous opening into the cavernous sinus of the aneurysm dome, trans-arterial balloon embolization of the CCF and trapping of the aneurysm [5-7,10-12]. Intervention for occlusion of the fistula and pseudoaneurysm are initiated at least one month after head injury. Except for one case, transarterial balloon embolization for intradural pseudoaneurysm is initiated 10 days after trauma. A life-threatening SAH occurs as a result of the rupture of the pseudoaneurysm 90 minutes after the intervention [11]. Mechanical damage of the aneurysmal wall by intravascular manipulation or increasing intramural pressure proximal to the inflated balloon could have led to rupture of the pseudoaneurysm [7]. In the present case, there is no existence of CCF for venous decompression of the pseudoaneurysm. Increased risk of rupture after occlusion of the pseudoaneurysm by intravascular manipulation is taken into account.

Histological confirmation is not obtained in our case. We consider our case as a traumatic pseudoaneurysm according to history of traumatic head injury, location far from the branching area, irregular outline, and absence of neck and rapid increased size of the aneurysm. Intra-operatively, avulsion of right side P-com artery is noted and then clipping of the proximal stump of the P-com is performed. After the operation, there is no delayed subarachnoid hemorrhage noted. Due to few cases reported in the literature, there is no definite method for managing traumatic pseudoaneurysm of P-com artery currently. Here, we presented the fourth case of P-com artery traumatic pseudoaneurysm. Early diagnosis and appropriate management under stable condition one month after the head injury might improve the condition of high morbidity and mortality.

\section{Conclusions}

We present the fourth case of P-com traumatic pseudoaneurysm. Delay diagnosis of supra-clinoid pseudoaneurysm is confirmed three 
Citation: Su HY, Su YF, Wang CJ (2015) A Rare Case of Posterior Communicating Artery Pseudoaneurysm Caused by Traumatic Head Injury. J Trauma Treat 4: 248. doi:10.4172/2167-1222.1000248

Page 4 of 4

weeks after head injury. Avulsion of P-com artery is noted intra-operatively. There is no delayed SAH noted after clipping the proximal stump of the avulsed P-com artery. We discuss the relationship between pseudoaneurysm and traumatic head injury. Early diagnosis and appropriate management of the catastrophic disease is very important.

\section{References}

1. Mao Z, Wang N, Hussain M, Li M, Zhang H, et al. (2012) Traumatic intracranial aneurysms due to blunt brain injury-a single center experience. Acta Neurochir (Wien) 154: 2187-2193

2. Dubey A, Sung WS, Chen YY, Amato D, Mujic A, et al. (2008) Traumatic intracranial aneurysm: a brief review. J Clin Neurosci 15: 609-612. Jun;15(6):609-12

3. Ohba S, Kuroshima Y, Mayanagi K, Inamasu J, Saito R, et al. (2009) Traumatic aneurysm of the supraclinoid internal carotid artery-case report-. Neurol Med Chir (Tokyo) 49: 587-589.

4. Horiuchi T, Nakagawa F, Miyatake M, Iwashita T, Tanaka Y, et al. (2007) Traumatic middle cerebral artery aneurysm: case report and review of the literature. Neurosurg Rev 30: 263-267.

5. Chen Y, Jiang DY, Tan HQ, Wang LH, Chen XY, et al. (2009) Successful Transarterial Guglielmi Detachable Coil Embolization of Post-Traumatic Fistula between a Posterior Communicating Artery Aneurysm and the Cavernous Sinus. A Case Report. Interv Neuroradiol 15: 435-441.
6. Tytle TL, Loeffler CL, Steinberg TA (1995) Fistula between a posterior communicating artery aneurysm and the cavernous sinus. AJNR Am J Neuroradiol. 16:1808-1810.

7. Fu Y, Ohata K, Tsuyuguchi N, Hara M (2002) Direct surgery for posttraumatic carotid-cavernous fistula as a result of an intradural pseudoaneurysm: case report. Neurosurgery. 51:1071-1073.

8. Chedid MK, Vender JR, Harrison SJ, McDonnell DE (2001) Delayed appearance of a traumatic intracranial aneurysm. Case report and review of the literature. J Neurosurg 94: 637-641.

9. Haji FA, Boulton MR, de Ribaupierre S (2011) Blister-like supraclinoid internal carotid artery pseudoaneurysm in a 15-year-old male: case report and review of the literature. Pediatr Neurosurg 47: 449-454.

10. Masana Y, Taneda M (1992) Direct approach to a traumatic giant interna carotid artery aneurysm associated with a carotid-cavernous fistula. Case report. J Neurosurg 76: 524-527.

11. Komiyama M, Yasui T, Yagura H, Fu Y, Nagata $Y$ (1991) Traumatic carotidcavernous sinus fistula associated with an intradural pseudoaneurysm: a case report. Surg Neurol 36: 126-132.

12. Reddy SV, Sundt TM Jr (1981) Giant traumatic false aneurysm of the internal carotid artery associated with a carotid-cavernous fistula. Case report. J Neurosurg 55: 813-818. 